\title{
Generalizations of tournaments: A survey
}

\author{
Jørgen Bang-Jensen* $\quad$ Gregory Gutin ${ }^{\dagger}$
}

March 9, 2003

\begin{abstract}
We survey results concerning various generalizations of tournaments. The reader will see that tournaments are by no means the only class of directed graphs with a very rich structure. We describe, among numerous other topics mostly related to paths and cycles, results on hamiltonian paths and cycles. The reader will see that although these problems are polynomially solvable for all of the classes described, they can be highly non-trivial, even for these "tournament-like" digraphs.
\end{abstract}

\section{Introduction}

Tournaments are no doubt the most well understood class of directed graphs. They have a very rich structure, in particular with respect to cycles and paths; see e.g. recent survey papers [16, 75]. Some of this rich structure comes from the simple fact that tournaments have no pair of non-adjacent vertices and at first glance it may seem as if this must be the case for most of the nice structure (knowing that for general digraphs very little is known about the structure of paths and cycles). However, it is not the case as one can see from some results on generalizations of tournaments discussed in this survey.

In [51], a surprisingly simple characterization of those semicomplete multipartite digraphs that have a hamiltonian path was obtained. This characterization implies that the hamiltonian path problem is tractable for this important class of digraphs that remained for a long time the only generalization of tournaments (other than the semicomplete digraphs ) to be studied. It was also known, see e.g. [55], that the hamiltonian cycle problem for semicomplete multipartite digraphs was quite difficult. A polynomial algorithm for this problem was recently found [22]. This algorithm is based on results from [85].

In [6] the class of locally semicomplete digraphs was introduced and it was proved that a number of structural properties of tournaments are still valid for this much larger class of digraphs. In particular the hamiltonian path and cycle problems are both easy for locally semicomplete digraphs : every connected (strongly connected, respectively) locally semicomplete digraph has a hamiltonian path (cycle, respectively). The introduction of locally semicomplete digraphs has stimulated a significant amount of work, whose purpose has been to discover and study classes of digraphs that contain the tournaments and share many of their properties. In particular two entire Ph. D. theses have been devoted to this project $[42,64]$. It is the purpose of this survey to report on the current status of the research on these recent generalizations of tournaments.

The reader will see that tournaments are far from being the only digraphs with a rich path and cycle structure. We will also show that, even though some of the classes introduced (e.g. extended semicomplete digraphs) may seem, at first glance, to be just one more generalization with no particular

\footnotetext{
* Department of Mathematics and Computer Science,Odense University, Denmark

†Dept. of Maths and Stats, Brunel University, Uxbridge, Middlesex UB8 3PH, U.K.
} 
importance of its own, this is by no means the case. In Section 8 (see also $[10,13,14]$ ) we show how the results on paths and cycles for many of these classes of generalizations of tournaments can be combined to show that the hamiltonian path and cycle problem and even the heaviest path and cycle problems (with respect to vertex weights) can be solved for some classes of digraphs that are quite general.

Apart from some very recent development on semicomplete multipartite digraphs we will not cover this class of digraphs since this was done recently in two survey papers $[55,84]$. We do however wish to refer the reader to a recent application of Yeo's results on semicomplete multipartite digraphs in [85] to a problem on hamiltonian cycles avoiding prescribed arcs in tournaments [20]. In this paper it is shown how one can obtain a highly non-trivial result on tournaments using a result on cycles in semicomplete multipartite digraphs. This is a good example showing that results on generalizations of tournaments may be useful even if one is only interested in tournaments themselves.

\section{Terminology}

We shall assume that the reader is familiar with standard terminology on directed graphs (see e.g. [33]). For a given digraph $D$ we use $V(D)(E(D))$ to denote the vertex set (arc set) of $D$. We always use $n$ $(m)$ to denote the number of vertices (arcs) in the digraph currently under consideration. Sometimes we will also use the notation $|D|$ for the number of vertices in $D$.

The underlying undirected graph $U(D)$ is the graph obtained from $D$ by omitting all orientations and removing multiple edges if any; $D$ is connected if $U(D)$ connected; $\alpha(D)$ denotes the size of a largest independent set of vertices in $U(D)$.

If there is an arc from $x$ to $y$ in the digraph $D$, then we say that $x$ dominates $y$ and we shall use the notation $x \rightarrow y$ to denote this; $x \rightarrow y$ also denotes an arc from $x$ to $y$. For a given vertex $v \in V(D)$ we use $N^{+}(v)\left(N^{-}(v)\right)$ to denote the set of out-neighbours (in-neighbours) of $v$ and let $d^{+}(v)=\left|N^{+}(v)\right|$, $d^{-}(v)=\left|N^{-}(v)\right|$. A digraph $D$ is $k$-diregular (or, just, diregular) if $d^{+}(v)=d^{-}(v)=k$ for every $v \in V(D)$. If $A$ and $B$ are disjoint subsets of $V(D)$, then we use the notation $A \Rightarrow B$ to denote the fact that $a \rightarrow b$ for all $a \in A$ and all $b \in B$ and there is no arc from $B$ to $A$.

For any subset $A$ of $V(D) \cup E(D), D-A$ denotes the subgraph obtained by deleting all vertices of $A$ and their incident arcs and then deleting the arcs of $A$ still present. If $A \subseteq V(D)$, then we let $D\langle A\rangle=D-(V(D)-A)$. We write $D-x$ instead of $D-\{x\}$ when $x \in V(D) \cup E(D)$.

Paths and cycles are always directed. A path from $x$ to $y$ is called an $(x, y)$-path. If $\mathrm{P}$ is a path containing a subpath from $x$ to $y$, then we let $P[x, y]$ denote the part of $\mathrm{P}$ from $x$ to $y$. A $k$-path-cycle subgraph, $k \geq 0$, is a collection of $k$ paths and some (possibly zero) cycles all vertex disjoint. The order of a $k$-path-cycle subgraph is the number of vertices that it covers. A $k$-path-cycle factor, $k \geq 0$, is a $k$ path-cycle subgraph that covers all the vertices of $D$. When $k=0$ we use the names cycle subgraph and cycle factor. Clearly, a cycle factor is a spanning 1-diregular subgraph. When a $k$-path-cycle subgraph (factor) contains no cycles we use the name $k$-path subgraph ( $k$-path factor).

A strong component $D^{\prime}$ of a digraph $D$ is a maximal subdigraph, such that for any two vertices $x, y \in D^{\prime}, D^{\prime}$ contains an $(x, y)$-path and a $(y, x)$-path. A digraph $D$ is strongly connected if it has only one strong component. $D$ is $k$-strongly connected if for any set $A$ of at most $k-1$ vertices, $D-A$ is strong.

An out-branching (in-branching) $T$ rooted at $v$ in a digraph is a spanning tree such that each $x \in V(T)-v$ has one arc coming in (going out). A digraph $D$ is transitive if, for any triple $x, y, z$ of distinct vertices of $D$ such that $x y$ and $y z$ are $\operatorname{arcs}$ of $D$, the $\operatorname{arc} x z$ is in $D$. Clearly, a transitive oriented graph has no cycle.

We finish this section by recalling two well-studied classes of graphs. A graph is a comparability graph if it can be oriented as a transitive digraph. A graph $G$ is a circular-arc graph if it can be represented 
by closed intervals (arcs) on a circle, i.e. each vertex corresponds to an interval on the circle and two vertices are adjacent just if their intervals have non-empty intersection. If the representation can be chosen such that no interval is contained in any other interval, then $G$ is a proper circular-arc graph.

\section{Various generalizations of tournaments}

In this section we define a number of classes of digraphs all of which are generalizations of tournaments.

A semicomplete digraph is a digraph with no non-adjacent vertices. A tournament is an oriented graph with no non-adjacent vertices. Thus tournaments are a special subclass of the semicomplete digraphs.

A digraph $D$ is locally in-semicomplete (locally out-semicomplete, respectively) if, for every vertex $x$ of $D$, the in-neighbourhood of $x$ (its out-neighbourhood, respectively) induces a semicomplete digraph. A digraph $D$ is locally semicomplete if it is both locally in- and locally out-semicomplete. Clearly every semicomplete digraph is locally semicomplete. Similarly we can define locally in-tournament digraphs, locally out-tournament digraphs and locally tournament digraphs by merely replacing the words "semicomplete" or semicomplete digraph" by "tournament" in the definitions above.

A digraph $D$ is a semicomplete $k$-partite digraph $(k \geq 2)$ or a semicomplete multipartite digraph if $U(D)$ is a complete $k$-partite graph.

A digraph $D$ is an arc-local tournament digraph if it satisfies that for every pair of adjacent vertices $x, y \in V(D)$ every vertex of $N^{+}(x)\left(N^{-}(x)\right)$ is adjacent to every vertex of $N^{+}(y)\left(N^{-}(y)\right)$.

A digraph $D$ is quasi-transitive if, for any triple $x, y, z$ of distinct vertices of $D$ such that $x y$ and $y z$ are $\operatorname{arcs}$ of $D$, there is at least one arc between $x$ and $z$. Clearly a semicomplete digraph is quasi-transitive. Note that if there is only one arc between $x$ and $z$, it can go in any direction, hence a quasi-transitive digraphs are generally not transitive.

A digraph $D$ is $k$-path-mergeable for some integer $k \geq 2$, if for any choice of vertices $x, y \in V(D)$ and any pair of internally disjoint $(x, y)$-paths $P, P^{\prime}$, each of length at most $k$, there exists an $(x, y)$ path $P^{*}$ in $D$, such that $V\left(P^{*}\right)=V(P) \cup V\left(P^{\prime}\right)$. A digraph $D$ of order $n$ is path-mergeable if it is $n$-path-mergeable.

The following very useful proposition is easy to prove.

Proposition 3.1 [9] Every locally in-semicomplete (out-semicomplete) digraph is path-mergeable.

Let $D$ be a digraph on $p$ vertices $v_{1}, \ldots, v_{p}$ and let $L_{1}, \ldots, L_{p}$ be a disjoint collection of digraphs. Then $D\left[L_{1}, \ldots, L_{p}\right]$ is the new digraph obtained from $D$ by replacing each vertex $v_{i}$ of $D$ by $L_{i}$ and adding an arc from every vertex of $L_{i}$ to every vertex of $L_{j}$ if and only if $v_{i} \rightarrow v_{j}$ is an arc of $D(1 \leq i \neq j \leq p)$.

Let $\Phi$ be a set of digraphs, we call the elements of $\Phi \quad \Phi$-graphs. A digraph $D$ is called an extended $\Phi$-graph if either it has only one vertex, or there is a decomposition $D=R\left[H_{1}, \ldots, H_{r}\right]$ such that $R \in \Phi$, each of the digraphs $H_{i}, i=1, \ldots, r$ has no arcs, and $r \geq 2$. A set $\Phi$ is extension-closed if each extended $\Phi$-graph is in $\Phi$. In particular, semicomplete multipartite digraphs are extension-closed, whereas the set of semicomplete digraphs is not extension-closed.

\section{Strong components}

The strong component digraph $S C(D)$ of a digraph $D$ is obtained by contracting each strong component of $D$ to a single vertex. The strong component digraph of a tournament is just a transitive tournament. 
The structure of strong component digraphs of generalizations of tournaments are not so simple but we still have some nice and useful properties here.

The following theorem on strong components in a non-strong extended locally semicomplete digraph generalizes the corresponding result for locally semicomplete digraphs [6].

Proposition 4.1 [14] Let D be a connected non-strongly connected extended locally semicomplete digraph.

1. If $A$ and $B$ are distinct strong components of $D$, then either $A \Rightarrow B$, or $B \Rightarrow A$, or there are no arcs between $A$ and $B$.

2. There is a unique ordering $D_{1}, \ldots, D_{s}, s \geq 2$ of the strong components of $D$ so that there is no arc from $D_{j}$ to $D_{i}$ for $j>i$.

3. Furthermore with this ordering we have $D_{1} \Rightarrow D_{2} \Rightarrow \ldots \Rightarrow D_{s}$.

When 2. holds for a digraph $D$, then we call the ordering $D_{1}, D_{2}, \ldots, D_{s}$ the strong decomposition of D.

Another kind of decomposition theorem for non-strong locally semicomplete digraphs was described in $[45]$.

Theorem 4.2 [45] Let $D$ be a connected locally semicomplete digraph that is not strongly connected and let $D_{1}, \ldots, D_{p}$ be the strong decomposition of $D$. Then $D$ can be decomposed in $r \geq 2$ subdigraphs $D_{1}^{\prime}, D_{2}^{\prime}, \ldots, D_{r}^{\prime}$ as follows:

$$
\begin{gathered}
D_{1}^{\prime}=D_{p}, \quad \lambda_{1}=p, \\
\lambda_{i+1}=\min \left\{j \mid N^{+}\left(D_{j}\right) \cap V\left(D_{i}^{\prime}\right) \neq \emptyset\right\}, \quad \text { and } \\
D_{i+1}^{\prime}=D\left\langle V\left(D_{\lambda_{i+1}}\right) \cup V\left(D_{\lambda_{i+1}+1}\right) \cup \cdots \cup V\left(D_{\lambda_{i}-1}\right)\right\rangle .
\end{gathered}
$$

The subdigraphs $D_{1}^{\prime}, D_{2}^{\prime}, \ldots, D_{r}^{\prime}$ satisfy the properties below:

(a) $D_{i}^{\prime}$ consists of some strong components of $D$ and is semicomplete for $i=1,2, \ldots, r$;

(b) $D_{i+1}^{\prime}$ dominates the initial component of $D_{i}^{\prime}$ and there exists no arc from $D_{i}^{\prime}$ to $D_{i+1}^{\prime}$ for $i=$ $1,2, \ldots, r-1$;

(c) if $r \geq 3$, then there is no arc between $D_{i}^{\prime}$ and $D_{j}^{\prime}$ for $i, j$ satisfying $|j-i| \geq 2$.

For a connected, but not strongly connected locally semicomplete digraph $D$, the unique sequence $D_{1}^{\prime}, D_{2}^{\prime}, \ldots, D_{r}^{\prime}$ defined in Theorem 4.2 is called the semicomplete decomposition of $D$.

Some properties of the strong components of a locally in-semicomplete digraph are described in the following two results from [27].

Theorem 4.3 Let $D$ be a locally in-semicomplete digraph.

(i) Let $A$ and $B$ be distinct strong components of $D$. If a vertex $a \in A$ dominates some vertex in $B$, then $a \Rightarrow B$. Furthermore $A \cap N^{-}(b)$ induces a semicomplete digraph for each $b \in B$. 
(ii) If $D$ is connected, then $S C(D)$ has an out-branching. Furthermore, if $R$ is the root of that outbranching and $A$ is any other component, there is a path from $R$ to $A$ containing all the components that can reach $A$.

Corollary 4.4 Let $D$ be a strongly connected locally in-semicomplete digraph and let $S$ be a minimal separating set (w.r.t inclusion). There is a unique order $D_{1}, \ldots, D_{k}$ of the strong components of $D-S$, such that there are no arcs from $D_{j}$ to $D_{i}$ for $j>i$ and $D_{i}$ has an arc to $D_{j}$ for $i=1, \ldots, k-1$.

The structure of the strong components of quasi-transitive digraphs is described in Theorem 6.1.

\section{Structure of underlying graphs and recognition}

We first discuss the question of which graphs can be the underlying graphs of the various classes of digraphs defined in Section 2 and the question of deciding whether a given digraph belongs to one of those classes.

It turns out that locally semicomplete digraphs and quasi-transitive digraphs have very well-known underlying graphs.

Theorem 5.1 [76] The underlying graphs of locally semicomplete digraphs are precisely the proper circular-arc graphs.

Theorem 5.2 [38] The underlying graphs of quasi-transitive digraphs are precisely the comparability graphs.

In a series of papers $[34,35,62]$ Hell, Huang and others have used this close relation between locally semicomplete digraphs and proper circular-arc graphs to devise fast algorithms for many problems for proper circular-arc graphs. See also [24].

The precise structure of the underlying graphs of locally in-semicomplete digraphs is currently not known. In [81] a characterization was given in terms of intersection graphs and a characterization by forbidden induced subgraphs was described. However, these obstructions seem difficult to recognize and the intersection graph characterization is not very useful for studying structural properties of locally in-semicomplete digraphs. Thus it would be useful to obtain other more 'structural' characterizations of the underlying graphs of locally in-semicomplete digraphs.

It was shown in [27] that all chordal and all circular-arc graphs can be oriented as locally insemicomplete digraphs. Furthermore a topological property of graphs orientable as locally in-semicomplete digraphs was obtained. It was also shown that every graph which can be represented in a uni-cyclic graph can be oriented as a locally in-semicomplete digraph. We refer the interested reader to [27].

For graphs that can be oriented as path-mergeable digraphs not much is known other than the fact that, by Proposition 3.1 every graph that is orientable as an locally in-semicomplete digraph can be oriented as path-mergeable digraph.

Problem 5.3 Characterize the underlying graphs of path-mergeable digraphs. Is there a polynomial algorithm to recognize these graphs?

It is not difficult to see that there is a polynomial algorithm to decide whether a given digraph is quasi-transitive, locally in-semicomplete, or locally out-semicomplete: the restriction is locally defined and concerns only two arcs. Thus, recognition of the three classes of digraphs above can be done in 
time $O(\Delta m)$, where $\Delta$ denotes the maximum degree of the underlying graph. Similarly, the underlying graphs of these classes can be recognized in time $O(\Delta m)$, using a reduction to 2-satisfiability (see [27, Theorem 5.1] for details). In [24] an optimal $O(n+m)$ algorithm to recognize locally semicomplete digraphs is given. This is based on Theorem 6.7 and an optimal $O(n+m)$ algorithm to recognize proper circular-arc graphs in $[34,35]$. It is worth noting that the algorithm in $[34,35]$ explicitly uses the fact that a graph is a proper circular-arc graph if and only if it can be oriented as a round locally tournament digraph (the definition of a round locally tournament digraph is given in the next section). Another $O(\Delta m)$ algorithm for recognizing comparability graphs was given in [62].

It is not quite as simple to see that path-mergeable digraphs are also recognizable in polynomial time, but using a more detailed analysis of their structure this is possible and an $O\left(\mathrm{~m}^{3}\right)$ recognition algorithm is described in [9].

\section{Structural characterizations}

The following theorem characterizes quasi-transitive digraphs in a recursive fashion.

Theorem 6.1 [25] A digraph $D$ is quasi-transitive if and only if the following holds.

(1) If $D$ is not strongly connected, then there exist a natural number $q \geq 2$, a transitive oriented graph $Q$ on $q$ vertices and strongly connected quasi-transitive digraphs $W_{1}, W_{2}, \ldots, W_{q}$ such that $D=Q\left[W_{1}, W_{2}, \ldots, W_{q}\right]$.

(2) If $D$ is strongly connected, then there exist a natural number $q \geq 2$, a strongly connected semicomplete digraph $Q$ on $q$ vertices and quasi-transitive digraphs $W_{1}, W_{2}, \ldots, W_{q}$, where each $W_{i}$ is either a single vertex or a non-strongly connected quasi-transitive digraph, such that $D=$ $Q\left[W_{1}, W_{2}, \ldots, W_{q}\right]$. Furthermore, if $Q$ has a cycle of length two induced by vertices $v_{i}$ and $v_{j}$, then the corresponding digraphs $W_{i}$ and $W_{j}$ are trivial, i.e., each of them has only one vertex.

Despite being much more general than semicomplete digraphs, quasi-transitive digraphs still have structure tightly linked to that of semicomplete digraphs. The above characterization has turned out to be very useful for proving results about quasi-transitive digraphs, see [10, 17, 25, 58].

For locally semicomplete digraphs there is also a very useful structure theorem and a very interesting relation between the different locally semicomplete digraphs with the same underlying graph. In order to explain these results we need some more definitions.

A digraph on $n$ vertices is round if we can label its vertices $v_{0}, v_{1}, \ldots, v_{n-1}$ so that for each $i$, $N^{+}\left(v_{i}\right)=\left\{v_{i+1}, \ldots, v_{i+d^{+}\left(v_{i}\right)}\right\}$ and $N^{-}\left(v_{i}\right)=\left\{v_{i-d^{-}\left(v_{i}\right)}, \ldots, v_{i-1}\right\}$ (all indices are modulo $n$ ). Note that every strongly connected round digraph is hamiltonian.

Alspach and Tabib characterized round tournaments [1] (they used the name "domination orientable", but we think the name round is more appropriate, since we are not reorienting the graph, but rather labeling its vertices). The first author extended this characterization to locally tournament digraphs (Theorem 6.2).

It was pointed out in [6] that the characterization in Theorem 6.2 does not extend to locally semicomplete digraphs. There are examples of round locally semicomplete digraphs where some vertex has a cycle in its out-neighbourhood.

Theorem 6.2 [6] A locally tournament digraph is round if and only if $N^{+}(v)$ and $N^{-}(v)$ induce transitive tournaments for all vertices $v \in V(D)$. 
Hence, if a locally tournament digraph $D$ is round, then there exists a unique (up to cyclic permutations) round labeling of $D$. We refer to this as the round labeling of $D$.

A locally semicomplete digraph $D$ is round decomposable if there exists a round locally tournament digraph $R$ on $r \geq 3$ vertices such that $D=R\left[S_{1}, \ldots, S_{r}\right]$, where each $S_{i}$ is a semicomplete digraph. We call $R\left[S_{1}, \ldots, S_{r}\right]$ a round decomposition of $D$.

Proposition 6.3 [11] If a locally semicomplete digraph $D$ is round decomposable, then it has a unique round decomposition $D=R\left[S_{1}, \ldots, S_{r}\right]$, where each $S_{i}$ is a strongly connected semicomplete digraph.

We shall call this the round decomposition of $D$ and whenever we use the phrase 'the round decomposition' below it is to be understood that we think of this decomposition.

Proposition 6.4 [11] There exists a polynomial algorithm to decide if a given locally semicomplete digraph $D$ has a round decomposition and find this decomposition if it exists.

In [11] a complete characterization of locally semicomplete digraphs is given. This characterization involves the structure of the strong components of a certain induced subgraph of the locally semicomplete digraph and is therefore not easy to state in a short form. Hence we shall only state the following easy corollary of the complete characterization of locally semicomplete digraphs in [11]. Already in this form the characterization is very useful; see e.g. $[10,11]$.

Theorem 6.5 [11] Let D be a locally semicomplete digraph. The exactly one of the following possibilities hold. Furthermore, given a locally semicomplete digraph $D$ we can decide in polynomial time which of the possibilities holds for $D$.

1. D is semicomplete and not round decomposable.

2. $D$ is round decomposable with a unique round decomposition $D=R\left[S_{1}, S_{2}, \ldots, S_{r}\right]$, where $R$ is a round locally tournament digraph on $r \geq 3$ vertices and each $S_{i}$ is a strongly connected semicomplete digraph.

3. $\alpha(D)=2$ and $D$ is not round decomposable.

Recall that $\alpha(D)$ is the independence number of $U(D)$.

Prior to Theorem 6.5 Huang [63] had given another characterization of locally semicomplete digraphs which implicitly contains the information given in Theorem 6.5. We state his main result below in a slightly reformulated way.

For a given proper circular-arc graph $G$ with a prescribed circular-arc representation we get a natural order on the vertices of $G$ by fixing a point on the circle and labeling the vertices $v_{1}, v_{2}, \ldots, v_{n}$ according to the clockwise ordering of the right endpoints of their intervals (circular arcs) on the circle with respect to this point. Since every proper circular-arc graph has a representation in which no two arcs cover the whole circle [39], we may assume that we are working with such a representation. Now it is not difficult to see that the following leads to a round local tournament orientation of $G$ : orient the edge between $v_{i}$ and $v_{j}$ from $v_{i}$ to $v_{j}$ just if the left endpoint of the $j$ th interval is contained in the $i$ th interval. Thus we have

Proposition 6.6 [6, 61, 63] Every proper circular-arc graph has an orientation as a round local tournament. Furthermore this orientation is unique up to a full reversal (reversing the orientation of all arcs). 
Theorem 6.7 [63] Let $G$ be a proper circular-arc graph, let $\bar{G}$ denote the complement graph of $G$ and let $C_{1}, \ldots, C_{k}$ denote the connected components of $\bar{G}$.

- If $\bar{G}$ is not bipartite, then $k=1$ and (up to a full reversal) $G$ has only one orientation as a locally tournament digraph, namely the round orientation.

- If $\bar{G}$ is bipartite then every orientation of $G$ as a locally tournament digraph can be obtained from the round locally tournament digraph orientation $D$ of $G$ by repeatedly applying one of the following operations:

1. reverse all arcs in $D$ that go between two different $C_{i}$ 's.

2. reverse all arcs in $D$ that lie inside some $C_{i}$.

It is also possible to derive a similar result for all orientations of $G$ as locally semicomplete digraphs. We refer the reader to [63] for the details.

Although locally in-semicomplete digraphs are much more general than locally semicomplete digraphs, we suspect that they have a nice structural characterization.

Problem 6.8 Find a non-trivial structural characterization of locally in-semicomplete digraphs.

\section{Longest paths and cycles in locally semicomplete digraphs, locally in-semicomplete digraphs, arc-local tournament di- graphs and their extensions}

By Camion and Redei's theorems, the longest cycle and path problems for tournaments are trivially transformed into the corresponding hamiltonian path and cycle problems, respectively. For general digraphs no such reduction exists.

The following generalization of Camion's theorem is due to Bang-Jensen, Huang and Prisner [27].

Theorem 7.1 Every strongly connected locally in-semicomplete digraph has a hamiltonian cycle.

Note that the last result allows us to transform the longest cycle problem for locally in-semicomplete digraphs to the hamiltonian cycle problem. In the same paper, the following characterization of locally in-semicomplete digraphs containing a hamiltonian path was obtained.

Theorem 7.2 [27] A locally in-semicomplete digraph has a hamiltonian path if and only if it contains a vertex that can be reached by all other vertices by a directed path.

The last theorem also allows us to transform the longest path problem for locally in-semicomplete digraphs to the hamiltonian path problem: find an in-branching of maximum order. Since every connected locally semicomplete digraph has an in-branching (by Proposition 4.1) we have

Theorem 7.3 [6] Every connected locally semicomplete digraph has a hamiltonian path.

Note the following generalization of Theorem 7.1 (compare with Proposition 3.1): 
Theorem 7.4 [9] A path-mergeable digraph $D$ is hamiltonian if and only if $D$ is strongly connected and $U[D]$ is 2-connected. There is an $O\left(n^{2} m\right)$-algorithm for finding a hamiltonian cycle in a hamiltonian path-mergeable digraph $D$.

The problem of deciding whether a path-mergeable digraph has a hamiltonian path seems much harder than that of deciding the existence of a hamiltonian cycle. This is because the path-merging property does not imply anything for paths with only one endvertex in common. For example, any orientation of a tree is a path-mergeable digraph, but among these only an undirected path oriented as (directed) path has a hamiltonian path. Also note that there exist infinitely many strongly connected path-mergeable digraphs without a hamiltonian path. For example, take four disjoint cycles $C_{1}, \ldots, C_{4}$ and identify a vertex from each of $C_{2}, C_{3}, C_{4}$ with different vertices of $C_{1}$. The resulting digraph is a strongly connected path-mergeable digraph and clearly has no hamiltonian path.

Problem 7.5 Characterize those path-mergeable digraphs that have a hamiltonian path. Is there a polynomial algorithm to decide the existence of such a path?

Bang-Jensen and Hell [23] studied the complexity of the longest path and cycle problems for locally in-semicomplete digraphs. They proved the following:

Theorem 7.6 Let $D$ be a locally in-semicomplete digraph with $n$ vertices and $m$ arcs. Given an inbranching of $D$ represented by lists of in-neighbours, one can find a hamiltonian path of $D$ in time $O(n \log n)$. Moreover, there is an $O(m+n \log n)$ algorithm for finding a longest path in $D$.

They also generalized an algorithm of Manoussakis [68] for tournaments and proved the following:

Theorem 7.7 There is an $O(m+n \log n)$ algorithm for finding a longest cycle in a strongly connected locally in-semicomplete digraph.

Obviously, for most digraphs strong connectivity is not sufficient to merit the existence of a hamiltonian cycle. It is natural to add another necessary condition: the digraph considered has a cycle factor. It is easy to check, in polynomial time, the existence of a such subgraph in a given (general) digraph and find one, if it exists, (see $[3,54,55,56]$ ) using any polynomial maximum matching algorithm (for bipartite graphs). In particular, we can do it in time $O\left(n^{2.5} / \sqrt{\log n}\right)$ applying the algorithm from [2].

Theorem 7.8 [14] An extended locally semicomplete digraph is hamiltonian if and only if it is strongly connected and has a cycle factor. Given a spanning cycle subgraph of an extended locally semicomplete digraph $D$, a hamiltonian cycle of $D$ can be found in time $O\left(n^{2}\right)$, where $n$ is the number of vertices in $D$.

Results analogous to Theorem 7.8 have been obtained for semicomplete bipartite digraphs [49, 60, 69], for extended semicomplete digraphs [56], for arc-local tournament digraphs [3], and for extended locally in-semicomplete digraphs [15]. We point out here that Theorem 7.8 does not hold for semicomplete multipartite digraphs as one can see from the examples in [19, 56] (see, also, [55]). Very recently the authors and A. Yeo gave a polynomial algorithm for the hamiltonian cycle problem in semicomplete multipartite digraphs.

Theorem 7.9 [22] There is an $O\left(n^{7}\right)$ algorithm to decide whether a given semicomplete multipartite digraph has a hamiltonian cycle and find one if it exists.

This algorithm is based on some sufficient conditions for semicomplete multipartite digraphs to contain a hamiltonian cycle obtained by Yeo in [85]. The fact that the hamiltonian cycle problem for 
semicomplete multipartite digraphs is polynomial time solvable suggests a possibility for the existence of a characterization of hamiltonian semicomplete multipartite digraphs. We would like to state this as a problem.

Problem 7.10 Find a characterization of hamiltonian semicomplete multipartite digraphs.

Yeo's sufficient conditions [85] are strengthenings of those obtained in [19]. While the conditions in [19] were mostly used to find short proofs of some known results on semicomplete multipartite digraphs, Yeo used his conditions to prove the following new results:

Theorem 7.11 Every diregular semicomplete multipartite digraph is hamiltonian.

Zhang [86] had conjectured that every diregular multipartite tournament is hamiltonian.

Theorem 7.12 Every $k$-strongly connected semicomplete multipartite digraph with at most $k$ vertices in each partite set is hamiltonian.

The last result was conjectured by Guo and Volkmann [82].

Theorem 7.8 can be generalized as follows.

Theorem 7.13 [14] Let D be a strongly connected extended locally semicomplete digraph. Given a cycle subgraph $F=C_{1} \cup \ldots \cup C_{t}$ of $D$ of maximum order one can find a (longest) cycle $C$ of $D$ such that $V(C)=V\left(C_{1}\right) \cup \ldots \cup V\left(C_{t}\right)$ in time $O\left(n^{2}\right)$.

Unlike Theorem 7.8, the last result is not true for semicomplete bipartite digraphs. We can obtain only a slightly weaker result.

Theorem 7.14 [56] Let $D$ be a strongly connected semicomplete bipartite digraph. Given a cycle subgraph $F=C_{1} \cup \ldots \cup C_{t}$ of $D$ of maximum order one can find a (longest) cycle $C$ of $D$ such that $|V(C)|=\left|V\left(C_{1}\right) \cup \ldots \cup V\left(C_{t}\right)\right|$ in time $O\left(n^{2}\right)$.

The difference between the last two theorems is reflected, in particular, in Theorem 8.5, where we deal with weights on the vertices.

Note that a cycle subgraph of maximum order of an arbitrary digraph $D$ can be found in time $O\left(n^{3}\right)$ solving an auxiliary assignment problem $[54,55,56]$.

Gutin [51] (see also $[19,54,55]$ ) found simple necessary and sufficient conditions for a semicomplete multipartite digraph to have a hamiltonian path. Analogous results are true for arc-local tournament digraphs [3] and extended locally semicomplete digraphs [14]. We formulate this result for the last family of digraphs.

Theorem 7.15 A connected extended locally semicomplete digraph $D$ has a hamiltonian path if and only if it contains a 1-path-cycle factor. Given a 1-path-cycle factor of D, one can construct a hamiltonian path of $D$ in time $O\left(n^{2}\right)$.

Note that, by Theorem 7.15, a hamiltonian path in an extended locally semicomplete digraph $D$ (if one exists) can be constructed in time $O\left(n^{2.5} / \sqrt{\log n}\right)$. Indeed, it is easy to see that a digraph $H$ has a 1path-cycle factor $F$ if and only if the digraph $H^{\prime}$, obtained from $H$ by adding a new vertex $x$ dominating 
and being dominated by every vertex of $H$, has a cycle factor. Hence, the problem of finding a 1-pathcycle factor is easily transformed to that for finding a cycle factor. The last problem was considered above.

Unlike Theorem 7.8, Theorem 7.15 cannot be generalized to extended locally in-semicomplete digraphs as one can see from the following example [15]. Define the extended locally in-semicomplete digraph $L$ on 6 vertices as follows:

$$
V(L)=\{1,2,3,4,5,6\}, A(L)=\{1 \rightarrow 2,2 \rightarrow 3,3 \rightarrow 4,4 \rightarrow 2,3 \rightarrow 1,3 \rightarrow 5,3 \rightarrow 6,5 \rightarrow 6,6 \rightarrow 5\} .
$$

$L$ contains a 1 -path-cycle factor consisting of a path $1 \rightarrow 2 \rightarrow 3 \rightarrow 4$ ) and a cycle $5 \rightarrow 6 \rightarrow 5$ (and even an in-branching rooted in the vertex 6 ), but has no hamiltonian path. It is natural to pose the following:

Problem 7.16 (1) Find a characterization of extended locally in-semicomplete digraphs containing hamiltonian paths;

(2) Find the complexity of the problem of deciding whether an extended locally in-semicomplete digraph has a hamiltonian path.

Theorem 7.15 can easily be generalized to longest paths.

Theorem 7.17 [14] The order of a longest path in an extended locally semicomplete digraph $D$ equals to the order of a 1-path-cycle subgraph of $D$ with maximum number of vertices. Moreover, given a 1 path-cycle subgraph $F$ of an extended locally semicomplete digraph $D$, a path $P$ such that $V(P)=V(F)$ can be found in time $O\left(n^{2}\right)$.

Results analogous to Theorem 7.17 hold for semicomplete multipartite digraphs [54] and arc-local tournament digraphs [3].

\section{Longest paths and cycles in quasi-transitive digraphs and their generalizations}

Bang-Jensen and Huang [25] characterized both quasi-transitive digraphs containing hamiltonian cycles and hamiltonian paths (Theorem 8.1) using the analogues of Theorems 7.8 and 7.15 for extended semicomplete digraphs as well as Theorem 6.1 .

Theorem 8.1 [25] Let D be a connected quasi-transitive digraph. Then

(1) $D$ is hamiltonian if and only if it is strongly connected and has a cycle factor $C_{1} \cup \ldots \cup C_{t}\left(C_{i}\right.$ is a cycle in $D, i=1,2, \ldots, t)$ such that there is no $C_{i}$ whose vertices is completely contained in a connected component of the complement of $U(D)$.

(2) $D$ contains a hamiltonian path if and only if it has a 1-path-cycle factor $P \cup C_{1} \cup \ldots \cup C_{t}, t \geq 0$ ( $P$ is a path and $C_{i}$ is a cycle in $\left.D, i=1, \ldots, t\right)$ such that neither $V(P)$ nor $V\left(C_{i}\right)$ is completely contained in a connected component of the complement of $U(D)$.

Bang-Jensen and Huang [25] noted that Theorem 8.1 does not seem to imply polynomial algorithms and conjectured that there exist such algorithms. In [58], Gutin developed an approach which solves the conjecture in affirmative. Below we describe basic aspects of this approach for more general, so-called totally $\Phi$-decomposable, digraphs. First, we define such digraphs. 
Let $\Phi$ be a set of digraphs containing the digraph with one vertex. A digraph $D$ is called $\Phi$ decomposable if either $D$ has only one vertex or there is a decomposition $D=H\left[S_{1}, \ldots, S_{h}\right], h \geq 2$ such that $H \in \Phi$ and $h=|H|$ (we call this decomposition a $\Phi$-decomposition). Note that every $\Phi$-graph is $\Phi$-decomposable: just take each $S_{i}$ as the graph with one vertex.

A digraph $D$ is called totally $\Phi$-decomposable if either $D \in \Phi$ or there is a $\Phi$-decomposition $D=$ $H\left[S_{1}, \ldots, S_{h}\right]$ such that $h \geq 2$, and each $S_{i}$ is totally $\Phi$-decomposable. In this case, a $\Phi$-decomposition of $D, \Phi$-decompositions $S_{i}=H_{i}\left[S_{i 1}, \ldots, S_{i h_{i}}\right]$ of all $S_{i}$ which have more than one vertex, $\Phi$-decompositions of those of $S_{i j}$ who has more than one vertex, and so on, form a set of digraphs which will be called a total $\Phi$-decomposition of $D$.

Let $\Phi_{0}$ be the union of all semicomplete multipartite, extended locally semicomplete and acyclic digraphs, $\Phi_{1}$ be the union of all extended locally semicomplete and acyclic digraphs, and $\Phi_{2}$ be the union of all semicomplete bipartite, extended locally semicomplete and acyclic digraphs. It was proved $[13,14]$ that in time $O\left(n^{4}\right)$, one can check if a given digraph $D$ is totally $\Phi_{i}$-decomposable $(i=0,1,2)$ and (in case it is so) construct a total decomposition of $D$. Note that $\Phi_{1} \subset \Phi_{2} \subset \Phi_{0}$. Moreover, every quasi-transitive digraph is totally $\Phi_{1}$-decomposable (by Theorem 6.1).

The first step of the approach in [58] is to generalize the hamiltonian path problem. The pathcovering number of a digraph $D$ (denoted by $p c(D)$ ) is the least positive integer $k$ such that $D$ has a $k$-path factor. The path-cycle-covering number of a digraph $D$ (denoted by $p c c(D))$ is the least positive integer $k$ such that $D$ has a $k$-path-cycle factor. The path-cycle-covering number of a digraph can easily be found in polynomial time using, in particular, algorithms on flows in networks [13, 17, 58]. The path-covering number is hard to calculate: note that $p c(D)=1$ iff $D$ has a hamiltonian path. Thus, the path-covering number problem generalizes the hamiltonian path problem.

For some digraphs $D, p c(D)=p c c(D)$. Fortunately, this is true for every $\Phi_{0}$-graph by Theorem 7.17 and its analogue for semicomplete multipartite digraphs. This fact leads to the solution of the path-covering number problem for quasi-transitive digraphs given below.

Let $D$ be a totally $\Phi_{0}$-decomposable digraph and $D=R\left[H_{1}, \ldots, H_{r}\right]$ be a $\Phi_{0}$-decomposition of $D$ (which is the part of a total $\Phi_{0}$-decomposition of $D$ ), and let $E_{p}$ be a digraph of order $p$ having no arcs. A $p c(D)$-path factor of $D$ passes through every $H_{i}$ several, say $n_{i}$, times. Clearly, $n_{i}$ satisfies the following inequality: $p c\left(H_{i}\right) \leq n_{i} \leq\left|H_{i}\right|$. Therefore,

$$
p c(D)=\min \left\{p c\left(R\left[E_{n_{1}}, \ldots, E_{n_{r}}\right]\right): p c\left(H_{i}\right) \leq n_{i} \leq\left|H_{i}\right|, i=1, \ldots, r\right\}
$$

Since $\Phi_{0}$ is extension-closed, and since, for every $\Phi_{0}$-graph $Q, p c(Q)=p c c(Q)$, we obtain

$$
p c(D)=\min \left\{p c c\left(R\left[E_{n_{1}}, \ldots, E_{n_{r}}\right]\right): p c\left(H_{i}\right) \leq n_{i} \leq\left|H_{i}\right|, i=1, \ldots, r\right\}
$$

The recursive formula (1) allows one to compute $p c(D)$ in polynomial time. To show that, it suffies to demonstrate how to find, in polynomial time, the minimum in formula (1) given all values of $p c\left(H_{i}\right)$ (and $\left|H_{i}\right|$ ). Construct a network $N_{R}$ containing the digraph $R$ and two additional vertices (source and sink) $s$ and $t$ such that $s$ and $t$ are adjacent to every vertex of $V(R)$ and the arcs between $s(t$, resp.) and $R$ are oriented from $s$ to $R$ (from $R$ to $t$, resp.). Associate with each vertex $v_{i}$ of $R$ (corresponding to $H_{i}$ in $D)$ the lower and upper bounds $p c\left(H_{i}\right)$ and $\left|V\left(H_{i}\right)\right|(i=1, \ldots, r)$ on the amount of flow that can pass through $v_{i}$. It is not difficult to see that the value of a minimum flow from $s$ to $t, m$, is related to the minimum in $(1)$, i.e. $p c(D)$, as follows: $p c(D)=\max \{1, m\}$. This, in particular, solves, in polynomial time, the hamiltonian path problem for quasi-transitive digraphs. For more details we refer the reader to $[58]$.

To solve, in polynomial time, the hamiltonian cycle problem for totally $\Phi_{2}$-decomposable digraphs, we shall use the following general proposition which can be justified analogously to formula (1). 
Proposition 8.2 Let $D=R\left[H_{1}, \ldots, H_{r}\right]$ be a decomposition of a digraph $D(r \geq 2)$. Then, $D$ is hamiltonian if and only if the following family $\mathcal{S}$ of digraphs contains a hamiltonian digraph:

$$
\mathcal{S}=\left\{R\left[E_{n_{1}}, \ldots, E_{n_{r}}\right]: p c\left(H_{i}\right) \leq n_{i} \leq\left|H_{i}\right|, i=1, \ldots, r\right\}
$$

Actually, if $D=R\left[H_{1}, \ldots, H_{r}\right]$ such that $R \in \Phi_{2}$ and every $H_{i}$ is totally $\Phi_{0}$-decomposable, then we can check whether $D$ is hamiltonian in polynomial time. Indeed, we can, in polynomial time, calculate all $p c\left(H_{i}\right)$ and $\left|H_{i}\right|$, verify whether $R$ is strongly connected, and check whether one of the members of $\mathcal{S}$ has a cycle factor (it has a cycle factor iff the network $N_{R}$ without $s$ and $t$ admits a circulation, i.e. a flow of value 0$)$. Note that a member of $\mathcal{S}$, which is a $\Phi_{2}$-graph, has a hamiltonian cycle iff it contains a cycle factor and it is strongly connected, i.e. $R$ is strongly connected (due to Theorem 7.8 and its analogue for semicomplete bipartite digraphs).

This means that the hamiltonian cycle problem is polynomial time solvable for totally $\Phi_{2}$-decomposable digraphs, in particular, for quasi-transitive digraphs. Since the analogue of Theorem 7.8 is not true for the class of semicomplete multipartite digraphs we cannot conclude that the hamiltonian cycle problem is polynomial time solvable for totally $\Phi_{0}$-decomposable digraphs. However, we suspect that this is the case.

Conjecture 8.3 The hamiltonian cycle problem is polynomial time solvable for totally $\Phi_{0}$-decomposable digraphs.

It is impossible to construct polynomial time algorithms for finding a longest path and a longest cycle in a quasi-transitive digraph using the approach above. Nevertheless, Alon (personal communication) conjectured that there exist polynomial algorithms for finding a longest path and a longest cycle in a quasi-transitive digraph. A new method allowing one to construct such algorithms was proposed by Bang-Jensen and Gutin [13, 17].

In the rest of the section, we assume that every digraph $D$ we consider has non-negative weights $w($.$) on the vertices. The weight w(H)$ of a subgraph of $D$ is the sum of the weights of its vertices. For a positive integer $k$, the symbol $w_{k}(D)$ denotes the weight of a heaviest $k$-path subgraph of $D$, i.e. one with the maximum weight among $k$-path subgraphs. For convenience we define $w_{0}(D)=0$. We consider the following problem called the HPS problem: Given a digraph $D$ on $n$ vertices, find a heaviest $k$-path subgraph of $D$ for every $k=1,2, \ldots, n$.

In [17], the following general theorem was obtained.

Theorem 8.4 Let $\Phi$ be a set of digraphs including the trivial digraph on one vertex. Suppose that $\Phi$ is extension-closed and, for every $D \in \Phi$ on $n$ vertices,

$$
w_{k+1}(D)-w_{k}(D) \leq w_{k}(D)-w_{k-1}(D)
$$

for each $k=1,2, \ldots, n-1$. If there is a constant $s \geq 2$ so that, for every $L \in \Phi$, the HPS problem can be solved in time $O\left(|V(L)|^{s}\right)$, then, for every totally $\Phi$-decomposable digraph $D$, the HPS problem can be solved in time $O\left(|V(D)|^{s+1}\right)$, provided we are given a total $\Phi$-decomposition of $D$.

Using Theorem 8.4 along with Theorems 7.13, 7.14,7.17 as well as the analogue of Theorem 7.17 for semicomplete multipartite digraphs, one can quite easily prove the following result.

Theorem 8.5 [14] Let $D$ be a digraph with nonnegative real weights on the vertices.

(1) If $D$ is totally $\Phi_{0}$-decomposable, then the HPS problem for $D$ can be solved in time $O\left(n^{5}\right)$; 
(2) If $D$ is totally $\Phi_{1}$-decomposable, then a maximum weight cycle of $D$ can be found in time $O\left(n^{5}\right)$;

(3) If $D$ is totally $\Phi_{2}$-decomposable, then a longest cycle of $D$ can be found in time $O\left(n^{5}\right)$.

The second part of Theorem 8.5 allows us to generalize a result from [19] on cycles through a given set of vertices in extended semicomplete digraphs.

Theorem 8.6 Let $D$ be a totally $\Phi_{1}$-decomposable digraph and $X$ be a non-empty subset of $V(D)$. Then one can check whether $D$ has a cycle through all the vertices of $X$ and find such a longest one (if it exists) in time $O\left(n^{5}\right)$.

This result follows from Theorem 8.5. Indeed, set $w(x)=n$ for every vertex $x$ in $X$ and $w(y)=1$ for every $y \notin X$, and find a maximum weight cycle $C$ in $D$. Obviously, $D$ has a cycle through $X$ iff $w(C) \geq n|X|$.

\section{Hamiltonian connectivity}

A digraph $D$ is strongly hamiltonian-connected (weakly hamiltonian-connected, resp.) if it has a hamiltonian $(x, y)$-path $((x, y)$-path or $(y, x)$-path, resp.) for every choice of distinct vertices $x, y \in V(D)$. Bang-Jensen, Guo and Volkmann [12] generalized Thomassen's characterization of weakly hamiltonian connected tournaments to locally semicomplete digraphs. To describe the main result of [12], we introduce a few families of locally semicomplete digraphs. The unique strongly connected tournament with four vertices is denoted by $T_{4}^{1}$. The semicomplete digraphs $T_{4}^{1}, T_{4}^{2}, T_{4}^{3}$, and $T_{4}^{4}$ are shown in Figure 1 in that order from left to right.

Figure 1: The digraphs $T_{4}^{1}, T_{4}^{2}, T_{4}^{3}$ and $T_{4}^{4}$.

We denote the set $\left\{T_{4}^{1}, T_{4}^{2}, T_{4}^{3}, T_{4}^{4}\right\}$ by $\mathcal{T}_{4}$. It is easy to see that every digraph of $\mathcal{T}_{4}$ has a unique hamiltonian cycle and no hamiltonian path between two vertices which are not consecutive on this hamiltonian cycle (such two vertices are called opposite).

Let $\mathcal{T}_{6}$ be the set of semicomplete digraphs with vertex set $\left\{x_{1}, x_{2}, a_{1}, a_{2}, a_{3}, a_{4}\right\}$, each member $D$ of $\mathcal{T}_{6}$ has a cycle $a_{1} \rightarrow a_{2} \rightarrow a_{3} \rightarrow a_{4} \rightarrow a_{1}$ and $D\left\langle\left\{a_{1}, a_{2}, a_{3}, a_{4}\right\}\right\rangle$ is isomorphic to one member of $\mathcal{T}_{4}$, in addition, $x_{i} \Rightarrow\left\{a_{1}, a_{3}\right\} \Rightarrow x_{3-i} \Rightarrow\left\{a_{2}, a_{4}\right\} \Rightarrow x_{i}$ for $i=1$ or $i=2$. Since every digraph of $\mathcal{T}_{4}$ has no hamiltonian path connecting any two opposite vertices, every digraph of $\mathcal{T}_{6}$ has no hamiltonian path between $x_{1}$ and $x_{2}$. It is straightforward to verify that $\mathcal{T}_{6}$ contains only two tournaments (denoted by $T_{6}^{\prime}$ and $\left.T_{6}^{\prime \prime}\right)$.

For every even integer $n \geq 4$ there is only one 2-strongly connected, 2-diregular locally semicomplete digraph (denoted by $R_{n}^{2}$ ) on $n$ vertices. We define

$$
\mathcal{T}^{*}=\left\{R_{n}^{2} \mid n \text { is even and } n \geq 4\right\} .
$$

We observe that every digraph of $\mathcal{T}^{*}$ has a unique hamiltonian cycle and is not weakly hamiltonian connected (see [7]). For instance, if the unique hamiltonian cycle of $R_{6}^{2}$ is denoted by $u_{1} \rightarrow u_{2} \rightarrow u_{3} \rightarrow u_{4} \rightarrow u_{5} \rightarrow u_{6} \rightarrow u_{1}$, then $u_{1} \rightarrow u_{3} \rightarrow u_{5} \rightarrow u_{1}$ and $u_{2} \rightarrow u_{4} \rightarrow u_{6} \rightarrow u_{2}$ are two cycles of $R_{6}^{2}$ and there is no hamiltonian path between any two vertices of $\left\{u_{1}, u_{3}, u_{5}\right\}$ or of $\left\{u_{2}, u_{4}, u_{6}\right\}$.

Let $T_{8}^{1}$ be the digraph consisting of $R_{6}^{2}$ together with two new vertices $x_{1}$ and $x_{2}$ such that

$$
x_{1} \Rightarrow\left\{u_{1}, u_{3}, u_{5}\right\} \Rightarrow x_{2} \Rightarrow\left\{u_{2}, u_{4}, u_{6}\right\} \Rightarrow x_{1} .
$$


Furthermore, $T_{8}^{2}\left(T_{8}^{3}\right.$, respectively) is defined as the digraph obtained from $T_{8}^{1}$ by adding the arc $x_{1} x_{2}$ (the arcs $x_{1} x_{2}$ and $x_{2} x_{1}$, respectively). We denote the set $\left\{T_{8}^{1}, T_{8}^{2}, T_{8}^{3}\right\}$ by $\mathcal{T}_{8}$. It is obvious that every element of $\mathcal{T}_{8}$ is a 3 -strongly connected locally semicomplete digraph and has also no hamiltonian path between $x_{1}$ and $x_{2}$.

Theorem 9.1 [12] A locally semicomplete digraph $D$ with at least three vertices is weakly hamiltonianconnected, if and only if it satisfies (i), (ii) and (iii) below:

(i) D is strongly connected,

(ii) the digraph $D-x$ has at most two strong components for each vertex $x$ of $D$,

(iii) $D$ is not isomorphic to any member of $\mathcal{T}_{6} \cup \mathcal{T}_{8} \cup \mathcal{T}^{*}$.

It is easy to see that the last theorem provides a polynomial algorithm for checking if a locally semicomplete digraph is weakly hamiltonian-connected. The proof of Theorem 9.1 in [12] is based on Theorem 6.5 and a complete characterization of those locally semicomplete digraphs that have two vertices $x$ and $y$ which are not connected by a hamiltonian path. This characterization generalizes $[78$, Theorem 2.1] see [12, Theorem 3.3].

Bang-Jensen, Gutin and Huang obtained the following characterization of weakly hamiltonianconnected extended tournaments.

Theorem 9.2 [18] Let $D$ be an extended tournament. $D$ is weakly hamiltonian-connected if and only if it satisfies (1), (2), (3), and (4) below.

(1) D is strongly connected.

(2) For every pair of distinct vertices $x$ and $y$ of $D$, there is a path $P$ connecting $x$ and $y$ so that $D-P$ has a cycle factor.

(3) For each vertex $x$ of $D, D-x$ has at most two strong components and, for each vertex $y$ in the initial (respectively, terminal) strong component, there is a $(y, x)$ (respectively, $(x, y)$ )-path $P^{\prime}$ such that $D-P^{\prime}$ has a cycle factor.

(4) D is not isomorphic to one of the two tournaments in $\mathcal{T}_{6}$.

An analogous characterization of weakly hamiltonian-connected semicomplete bipartite digraphs without 2-cycles was obtained by Bang-Jensen and Manoussakis [28]. The only difference between these two characterizations is in Condition 4: in Bang-Jensen's and Manoussakis' theorem the set of forbidden digraphs is absolutely different and moreover infinite.

In the case of strongly hamiltonian-connectivity no characterization is known even for the case of tournaments. Thomassen proved that every 4-strongly connected semicomplete digraph is strongly hamiltonian-connected and gave an infinite family of 3-strongly connected tournaments which are not strongly-hamiltonian connected [78]. Recently Guo extended this to locally semicomplete digraphs. His main result is the following.

Theorem 9.3 [44] Let $D$ be a 2-strongly connected locally semicomplete digraph and let $x, y$ be two distinct vertices of $D$. Then $D$ contains a hamiltonian path from $x$ to $y$ if $(a)$ or $(b)$ below is satisfied.

(a) The digraph $D$ has two internally disjoint $(x, y)$-paths $P_{1}, P_{2}$, each of which is of length at least 2 and a path $P$ which either starts in $x$, or ends in $y$ and has only $x$ or $y$ in common with $P_{1}, P_{2}$ such that $V(D)=V\left(P_{1}\right) \cup V\left(P_{2}\right) \cup V(P)$. Furthermore, for any vertex $z \notin V\left(P_{1}\right) \cup V\left(P_{2}\right)$, $z$ has a neighbour on $P_{1}-\{x, y\}$ if and only if it has a neighbour on $P_{2}-\{x, y\}$. 
(b) There are three internally disjoint $(x, y)$-paths in $D$, each of which is of length at least 2 and $D$ is not isomorphic to any of the first two members of $\mathcal{T}_{8}$.

In [42] Guo used this theorem to give a complete characterization of those 3-strongly connected arc3 -cyclic locally tournament digraphs with no hamiltonian path from $x$ to $y$ for specified vertices $x$ and $y$ (the definition of arc-3-cyclic digraphs is given in the next section). In particular this characterization shows that there exist infinitely many 3-strongly connected digraphs which are locally tournament digraphs but not tournaments and are not strongly hamiltonian-connected.

In [29] a polynomial algorithm for deciding whether a given semicomplete digraph has a hamiltonian path with specified initial and terminal vertices was described. It is interesting to note that this algorithm cannot be easily modified to solve the problem of finding the longest path with specified initial and terminal vertex in a semicomplete digraph. There also does not seem to be any simple reduction of this problem to the problem of deciding the existence of a hamiltonian path from $x$ to $y$.

Conjecture 9.4 There exists a polynomial algorithm which given a semicomplete digraph $D$ and two distinct vertices $x$ and $y$ of $D$ finds a longest $(x, y)$-path.

We believe that the result in [29] can be generalized to extended semicomplete digraphs and locally semicomplete digraphs.

Conjecture 9.5 There exists a polynomial algorithm which given an extended semicomplete digraph (a locally semicomplete digraph, resp.) $D$ and two distinct vertices $x$ and $y$ of $D$ decides whether $D$ has a hamiltonian path from $x$ to $y$ and finds such a path if one exists.

In conclusion we give the following natural generalization of a result by Thomassen [78] which has been used a number of times for solving problems concerning paths and cycles in tournaments.

Proposition 9.6 [9] Let D be a path-mergeable digraph. Then D has a hamiltonian ( $x, y)$-path if and only if $D$ contains a spanning acyclic digraph $H$ with a source $x$ and a sink $y$ so that, for every vertex $z \in V(D), H$ contains an $(x, z)$-path and $a(z, y)$-path.

\section{Pancyclicity and its generalizations}

A digraph $D$ is pancyclic if it has cycles of all lengths $3,4, \ldots, n$. D is vertex-pancyclic (arc-pancyclic, respectively) if for any $v \in V(D)(a \in E(D)$, respectively) and any $k \in\{3, \ldots, n\}$ there is a cycle of length $k$ containing $v$ ( $a$, respectively). $D$ is called arc-3-cyclic if each arc of $D$ is contained in a cycle of length 3. Let $x, y$ be a pair of distinct vertices of $D$. We say that $D$ has an $(x, y)$-path system if $D$ contains both an $(x, y)$-path of length $k$ and a $(y, x)$-path of length $k$ for every $k=2,3, \ldots, n-1$.

Gutin [57] (see, also, [55]) characterized pancyclic and vertex-pancyclic extended semicomplete digraphs. Bang-Jensen and Huang [25] reformulated this theorem (see below) and, using the result, found characterizations of pancyclic and vertex pancyclic quasi-transitive digraphs. They introduced the following notion. A digraph $D$ is triangular with partition $V_{0}, V_{1}, V_{2}$, if the vertex set of $D$ can be partitioned into three disjoint sets $V_{0}, V_{1}, V_{2}$ with $V_{0} \Rightarrow V_{1} \Rightarrow V_{2} \Rightarrow V_{0}$.

Theorem 10.1 [55, 57] Let $D$ be a hamiltonian extended semicomplete digraph with $k$ partite sets $(k \geq 3)$ of order $n \geq 4$.

(i) $D$ is pancyclic if and only if $D$ is not triangular with a partition $V_{0}, V_{1}, V_{2}$, two of which induce independent sets, such that either $\left|V_{0}\right|=\left|V_{1}\right|=\left|V_{2}\right|$ or no $D\left\langle V_{i}\right\rangle(i=0,1,2)$ contains a path of length 2 . 
(ii) $D$ is vertex-pancyclic if and only if it is pancyclic and either $k>3$ or $k=3$ and $D$ contains two cycles $Z, Z^{\prime}$ of length 2 such that $Z \cup Z^{\prime}$ has vertices in the three partite sets.

The last theorem extends the well-known results of Moser and Moon that every strongly connected tournament is both pancyclic and vertex-pancyclic.

Theorem 10.2 [25] Let $D$ be a hamiltonian quasi-transitive digraph on $n \geq 4$ vertices.

(i) $D$ is pancyclic if and only if it is not triangular with a partition $V_{0}, V_{1}, V_{2}$, two of which induce independent sets, such that either $\left|V_{0}\right|=\left|V_{1}\right|=\left|V_{2}\right|$, or no $V_{i}(i=0,1,2)$ contains a path of length 2.

(ii) $D$ is not vertex-pancyclic if and only if $D$ is not pancyclic or $D$ is triangular with a partition $V_{0}, V_{1}, V_{2}$ such that one of the following occurs:

1. $\left|V_{1}\right|=\left|V_{2}\right|$, both $D\left\langle V_{1}\right\rangle$ and $D\left\langle V_{2}\right\rangle$ are independent sets, and there exists a vertex $x \in V_{0}$ such that $x$ is not contained in any path of length 2 in $D\left\langle V_{0}\right\rangle$ (in which case $x$ is not contained in a cycle of length 5).

2. one of $D\left\langle V_{1}\right\rangle$ and $D\left\langle V_{2}\right\rangle$ is an independent set and the other contains no path of length 2, and there exists a vertex $x \in V_{0}$ such that $x$ is not contained in any path of length 1 in $D\left\langle V_{0}\right\rangle$ (in which case $x$ is not contained in a cycle of length 5).

Using Theorem 6.5 the following characterization of pancyclic and vertex-pancyclic locally semicomplete digraphs was derived in [11]. Below we use $g(R)$ to denote the the length of a shortest directed cycle in $R$ and $g_{r_{i}}(R)$ to denote the length of a shortest directed cycle through the vertex $r_{i} \in V(R)$. Note that, in particular, it follows from Theorem 10.3 that any strongly connected locally semicomplete digraph which is not vertex-pancyclic must be round decomposable.

Theorem 10.3 A strongly connected locally semicomplete digraph $D$ is pancyclic if and only if it is not of the form $D=R\left[S_{1}, \ldots, S_{r}\right]$, where $R$ is a round locally tournament digraph with $g(R)>$ $\max \left\{2,\left|V\left(S_{1}\right)\right|, \ldots,\left|V\left(S_{r}\right)\right|\right\}+1 . \quad D$ is vertex pancyclic if and only if $D$ is not of the form $D=$ $R\left[S_{1}, \ldots, S_{r}\right]$, where $R$ is a round locally tournament digraph with $g_{r_{i}}(R)>\max \left\{2,\left|V\left(S_{i}\right)\right|\right\}+1$ for some $i \in\{1, \ldots, r\}$, where $r_{i}$ is the vertex of $R$ corresponding to $S_{i}$.

Problem 10.4 Characterize arc-pancyclic locally semicomplete digraphs.

Note that even for semicomplete digraphs this problem is still open.

Problem 10.5 Characterize pancyclic (vertex-pancyclic, arc-pancyclic, resp.) locally in-semicomplete digraphs.

$\mathrm{Bu}$ and Zhang [31] obtained the following characterization of arc-pancyclic locally tournament digraphs.

Theorem 10.6 Let $T$ be connected, arc-3-cyclic locally tournament digraph. Then $T$ is arc-pancyclic if and only if $T$ is not isomorphic to $T_{8}^{2}$ (a member of the set $\mathcal{T}_{8}$ introduced in the previous section) or to a $\mathcal{D}_{6}$-type digraph or to $\mathcal{D}_{8}$-type digraph (see Figure 2). In particular, at most one edge of an arc-3-cyclic locally tournament digraph is not in cycles of all possible lengths. 

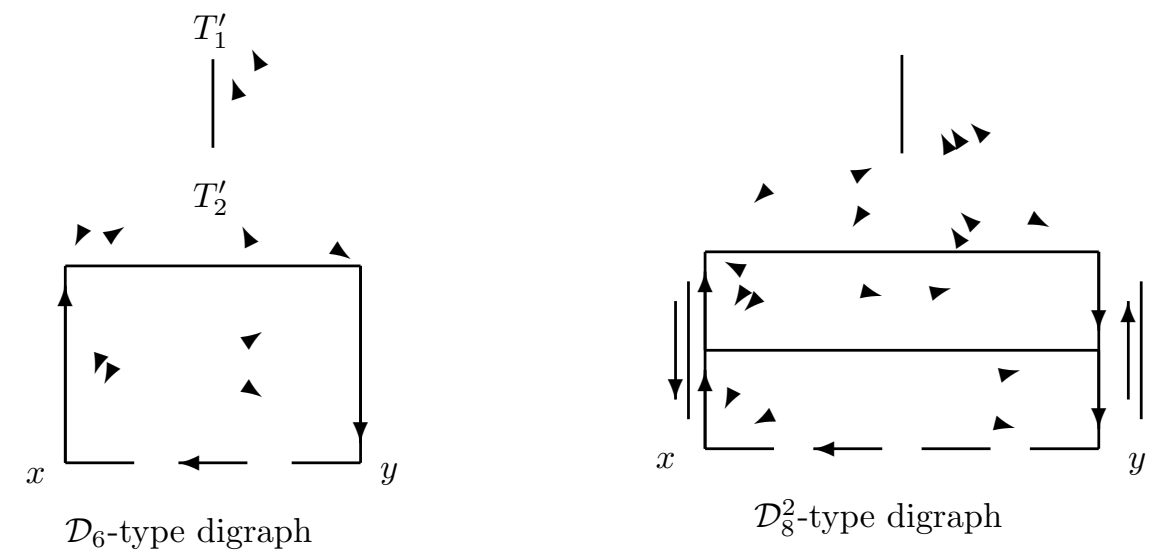

Figure 2: The arc from $y$ to $x$ can be left out and edges with no orientation may be oriented arbitrarily

The following two theorems in [41] deal with the existence of $(x, y)$-path systems.

The first theorem provides further properties of all but one arc-pancyclic locally tournament digraphs. A relatively short proof of this theorem together with Theorem 10.6 is given by Guo in [41].

Theorem 10.7 An arc-pancyclic locally tournament digraph $D$ contains an $(x, y)$-path system for every pair of non-adjacent vertices $x, y$ if and only if $D$ is not isomorphic to $T_{8}^{1}$ (a member of the set $\mathcal{T}_{8}$ introduced in the previous section).

A $\mathcal{D}_{3}$-type digraph $D$ consists of two vertex disjoint arc-3-cyclic tournaments $T^{\prime}$ and $T^{\prime \prime}$ and another vertex $x$ such that $x \Rightarrow V\left(T^{\prime}\right) \Rightarrow V\left(T^{\prime \prime}\right) \Rightarrow x$.

Theorem 10.8 Let $D$ be connected, arc-3-cyclic locally tournament digraph such that, for every arc $x \rightarrow y, D$ contains a vertex $z$ which dominates $y$ and is dominated by $x$. Then $D$ has an $(x, y)$-path system for pair $x, y$ of distinct vertices if and only if $D$ is not isomorphic to a $\mathcal{D}_{3}$-type digraph or to a $\mathcal{D}^{\infty}{ }_{8}$-type digraph (see Figure 3) or to one member of $\left\{T_{8}^{1}, T_{8}^{2}\right\}$ (see the definition of $\mathcal{T}_{8}$ given in the previous section).

In [40] Guo studied 3-strongly 3-arc-connected locally tournament digraphs. He characterized when such a locally tournament digraph $D$ does not have $(x, y)$-paths of all lenghts $3 \leq k \leq|D|-1$ for specified vertices $x, y$ satisfying that there is no arc from $y$ to $x$ in $D$. We refer the reader to [40] for the characterization.

One consequence of Guo's characterization is the following:

Theorem 10.9 [40] Let $D$ be a 5-strongly connected 3-arc-cyclic locally tournament digraph. Then the following is true

1. $D$ is arc-pancyclic.

2. For every arc $x \rightarrow y D$ contains $(x, y)$-paths of all lengths $3 \leq k \leq|D|-1$.

3. For every pair of non-adjacent vertices $x$ and $y D$ has an $(x, y)$-path system. 


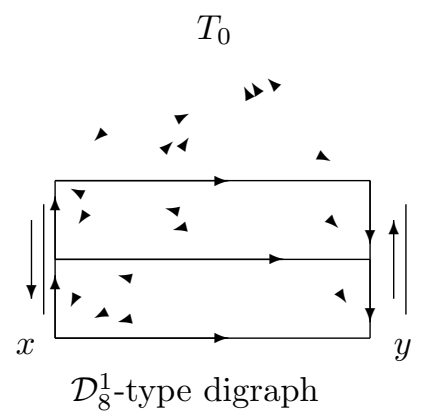

Figure 3: $T_{0}$ is an arc-3-cyclic tournament

\section{Disjoint paths}

Recall two classical NP-complete problems for directed graphs:

Problem 11.1 (The 2-path problem) Given a digraph $D$ and distinct vertices $u, v, x, y \in V(D)$. Decide if $D$ has a pair of vertex-disjoint paths $P_{u, v}, P_{x, y}$ where $P_{u, v}\left(P_{x, y}\right)$ is a $(u, v)$-path $((x, y)$-path).

Problem 11.2 (The weak 2-path problem) Given a digraph $D$ and distinct vertices $u, v, x, y \in$ $V(D)$. Decide if $D$ has a pair of arc-disjoint paths $P_{u, v}, P_{x, y}$ where $P_{u, v}\left(P_{x, y}\right)$ is a $(u, v)$-path $((x, y)$ path).

Theorem 11.3 [30] The 2-path problem is polynomially solvable for semicomplete digraphs.

Theorem 11.4 [5] The weak 2-path problem is polynomially solvable for semicomplete digraphs.

Using Theorem 6.1 it was shown in [10] that for quasi-transitive digraphs both problems can be reduced to the semicomplete case.

Theorem 11.5 [10] The 2-path problem and the weak 2-path problem are polynomially solvable for quasi-transitive digraphs.

In fact the following much more general results were shown in [10]:

Theorem 11.6 [10] The 2-path problem and the weak 2-path problem is polynomially solvable for all digraphs that can be obtained from strong semicomplete digraphs by substituting arbitrary digraphs for vertices.

Theorem 11.7 [10] Let $\Phi$ be a class of strongly connected digraphs, let $\Phi_{\text {ext }}$ denote the class of all extensions of graphs in $\Phi$ and let

$$
\Phi^{*}=\left\{F\left[D_{1}, \ldots, D_{|F|}\right]: F \in \Phi \text {, each } D_{i} \text { is a general digraph }\right\} .
$$

There is a polynomial algorithm for the 2-path problem in $\Phi^{*}$ if and only if there is a polynomial algorithm for the 2-path problem for all digraphs in $\Phi_{\text {ext }}$. 
This result shows again that studying extensions of digraphs can be quite useful.

It is important to note here that $\Phi$ must consist of strongly connected digraphs, since it is not difficult to reduce the 2-path problem for general digraphs (which is NP-complete [36]) to the 2-path problem for those digraphs that can be obtained from the digraph $H$ consisting of just an arc $u \rightarrow v$ by substituting arbitrary digraphs for the vertex $v$.

Corollary 11.8 [10] The 2-path problem and the weak 2-path problem are polynomially solvable for extended semicomplete digraphs.

Conjecture 11.9 The weak 2-path problem and the 2-path problem are polynomially solvable for locally semicomplete digraphs.

For semicomplete multipartite digraphs these problems seem quite difficult, but we suspect that these problems are tractable at least for some subclasses of semicomplete multipartite digraphs.

Conjecture 11.10 The 2-path problem and the weak 2-path problem are polynomially solvable for semicomplete bipartite digraphs.

A digraph $D$ is $k$-linked if for any set of $2 k$ distinct vertices $x_{1}, x_{2}, \ldots, x_{k}, y_{1}, y_{2}, \ldots, y_{k}, D$ contains $k$ vertex-disjoint paths $P_{1}, P_{2}, \ldots, P_{k}$ such that $P_{i}$ is an $\left(x_{i}, y_{i}\right)$-path.

The following result extends a result by Thomassen [79] to locally semicomplete digraphs and quasitransitive digraphs.

Theorem 11.11 [10] For every natural number $k$ there exists a natural number $g(k)(h(k))$ such that every $g(k)$-strongly connected locally semicomplete digraph ( $h(k)$-strongly connected quasi-transitive digraph) is k-linked.

Conjecture 11.12 For every natural number $k$ there exists a natural number $f(k)$ such that every $f(k)$-strongly connected semicomplete multipartite digraph is $k$-linked.

Even the case $k=2$ of Conjecture 11.12 seems highly non-trivial.

Consider now the problem of finding internally vertex-disjoint (arc-disjoint) $(x, y)-,(y, z)$-paths in a digraph. This problem is easily seen to be NP-complete, since there is a trivial reduction of the 2-path problem to this problem. It was shown in [10] that in the case of arc-disjoint paths the characterization for semicomplete digraphs given in [5] still holds for quasi-transitive digraphs and locally in-semicomplete digraphs.

Theorem 11.13 [10] Let $D$ be a digraph which is either a quasi-transitive digraph, or an locally insemicomplete digraph and let $x, y, z \in V(D)$ be distinct vertices. D has a pair of arc-disjoint $(x, y)$-, $(y, z)$-paths if and only if there is no arc in $E(D)$ that lies on all $(x, y)$-paths and on all $(y, z)$-paths. Furthermore there is a polynomial algorithm to find the desired paths if they exist.

In the case of internally vertex-disjoint paths (i.e. when we want an $(x, z)$-path that passes through $y$ ) it was pointed out in [10] that the complete characterization for semicomplete digraphs given in [4] is also valid for quasi-transitive digraphs. Hence it follows that this problem is polynomially solvable for quasi-transitive digraphs. We refer the reader to $[4,10]$ for the details. 


\section{Arc-disjoint in- and out-branchings}

Problem 12.1 Given a digraph $D$ and vertices $u, v$ (not necessarily distinct). Decide whether $D$ has a pair of arc-disjoint branchings $F_{u}^{+}, F_{v}^{-}$such that $F_{u}^{+}$is an out-branching rooted at $u$ and $F_{v}^{-}$is an in-branching rooted at $v$.

For general digraphs Problem 12.1 is NP-complete [5]. However, in [5] the problem was solved for the case of tournaments and the result implies the existence of a polynomial algorithm to find the desired branchings if they exist. In [25] it was shown that provided $u=v$ and $v$ is adjacent to all other vertices, the problem is still polynomially solvable, even for general digraphs, and the following holds:

Theorem 12.2 [25] Let $D$ be a strongly connected digraph and $v$ a vertex of $D$ such that $V(D)=$ $\{v\} \cup I(v) \cup O(v)$. Let $A=\left\{A_{1}, A_{2}, \ldots A_{k}\right\}\left(B=\left\{B_{1}, B_{2}, \ldots, B_{r}\right\}\right)$ denote the set of terminal (initial) components in $D\langle O(v)\rangle(D\langle I(v)\rangle)$. Then $D$ contains a pair of arc-disjoint branchings $F_{v}^{+}, F_{v}^{-}$such that $F_{v}^{+}$is an out-branching rooted at $v$ and $F_{v}^{-}$is an in-branching rooted at $v$ if and only if there exist two disjoint arc sets $E_{A}, E_{B} \subset E(D)$ such that all arcs in $E_{A} \cup E_{B}$ go from $O(v)$ to $I(v)$ and every component in $A_{i} \in A\left(B_{j} \in B\right)$ is incident with an arc from $E_{A}\left(E_{B}\right)$. Furthermore, there exists a polynomial algorithm to find the desired branchings, or demonstrate the non-existence of such branchings.

It was also shown in [25] that for quasi-transitive digraphs and $u=v$ the problem can be reduced to the case when $v$ is adjacent to all other vertices.

Proposition 12.3 [25] A quasi-transitive digraph $D$ contains arc-disjoint branchings $F_{v}^{+}, F_{v}^{-}$rooted at a vertex $v$ if and only if $D^{\prime}=D(\{v\} \cup I(v) \cup O(v))$ has arc-disjoint branchings $F_{v}^{\prime+}, F_{v}^{\prime-}$ rooted at $v$.

Problem 12.4 Characterize those locally semicomplete digraphs $D$ that have arc-disjoint branchings $F_{v}^{+}, F_{v}^{-}$for a given vertex $v \in V(D)$.

Conjecture 12.5 The arc-disjoint in and out-branching problem is polynomially solvable for locally semicomplete digraphs.

The following much harder problem may also be tractable.

Problem 12.6 Characterize those locally semicomplete digraphs (respectively, quasi-transitive digraphs) $D$ that have arc-disjoint branchings $F_{u}^{+}, F_{v}^{-}$for given vertices $u, v \in V(D)$.

This problem is open even for semicomplete digraphs, since the characterization given in [5] is only valid for tournaments.

Problem 12.7 Characterize those quasi-transitive digraphs $D$ that have arc-disjoint branchings $F_{u}^{+}, F_{v}^{-}$ for given vertices $u, v \in V(D)$.

Conjecture 12.8 Problem 12.1 is polynomially solvable for quasi-transitive digraphs.

Recall that this was shown in [25] for the special case when $u=v$.

A digraph $D$ is $k$-strongly arc-connected if $D-B$ is strongly connected for every set $B$ of at most $k-1$ arcs in $D$. 
Conjecture 12.9 [80] There exists a natural number $N$ such that every digraph $D$ which is $N$-strongly arc-connected has arc-disjoint branchings $F_{v}^{+}, F_{v}^{-}$for every choice of $v \in V(D)$.

We believe that something much stronger holds for tournaments:

Conjecture 12.10 There exists a function $f: N \rightarrow N$ such that for every natural number $k$ every $f(k)$ strongly arc-connected tournament $T$ has $2 k$ arc-disjoint branchings $F_{v, 1}^{+}, \ldots, F_{v, k}^{+}, F_{v, 1}^{-}, \ldots, F_{v, k}^{-}$such that $F_{v, 1}^{+}, \ldots, F_{v, k}^{+}$are out-branchings rooted at $v$ and $F_{v, 1}^{-}, \ldots, F_{v, k}^{-}$are in-branchings rooted at $v$, for every vertex $v \in V(T)$.

It was shown in [5] that $f(1)=2$.

\section{Complementary cycles}

Two cycles $C$ and $C^{\prime}$ of a digraph $D$ are complementary if $V(D)=V(C) \cup V\left(C^{\prime}\right)$. A digraph $D$ is cycle-complementary if $D$ has two complementary cycles, each of which has length at least 3 . Let $\gamma$ be an integer satisfying $3 \leq \gamma \leq \frac{|D|}{2}$. If for each integer $t$ with $\gamma \leq t \leq|D|-\gamma$, the digraph $D$ contains complementary cycles of lengths $t$ and $|D|-t$ respectively, then $D$ is complementary $\gamma$-pancyclic. In the special case $\gamma=3$ we call $D$ complementary pancyclic. Answering a special case of a question of Thomassen (see [74]) and building upon a partial result by Reid [74], Song [77] proved that except for one tournament on 7 vertices, all 2-strongly connected tournaments are complementary pancyclic.

The kth power of a cycle $C$ is the digraph obtained from $C$ by adding an arc from $x \in V(C)$ to $y \in V(C)$ if and only if the length of the $(x, y)$-path contained in $C$ is less than or equal to $k$. Hence the 1st power of $C$ is $C$ itself.

Theorem 13.1 [46] A 2-strongly connected locally semicomplete digraph on at least 8 vertices is cycle complementary if and only if it is not the second power of an odd cycle.

Problem 13.2 [42] Let $k$ be a positive integer. What is the least integer $f(k)$ such that all but a finite number of $f(k)$-strongly connected locally semicomplete digraphs can be decomposed into $k$ vertex disjoint cycles, each of which is of length at least 3?

Conjecture 13.3 [42] Let $k$ be a positive integer and let $D$ be a $k$-strongly connected locally semicomplete digraph on at least $3 k$ vertices. Then $D$ can be decomposed in $k$ vertex disjoint cycles, each of which is of length at least 3, unless $D$ is a member of a finite family of $k$-strongly connected locally semicomplete digraphs .

Guo and Volkmann generalized Songs' result for tournaments [77] to locally semicomplete digraphs. Recall that for a digraph $D$ we use the notation $g(D)$ to denote the length of a shortest directed cycle of length at least 3 in $D$

Theorem 13.4 [47] Let D be a 2-strong locally semicomplete digraph on at least 6 vertices, then $D$ is complementary $g(D)$-pancyclic, unless $D$ is the second power of an odd cycle, or one of 6 specific locally semicomplete digraphs (we refer the reader to [47] for the definition of these).

In [42] Guo posed the following problem which generalizes a question of Song for tournaments: 
Problem 13.5 [42] Let $k$ be a positive integer. What is the least integer $h(k)$ such that all but a finite number of $h(k)$-strongly connected locally semicomplete digraphs on $n$ vertices can be decomposed into $k$ vertex disjoint cycles of lengths $n_{1}, n_{2}, \ldots, n_{k}$, where $n_{1}, n_{2}, \ldots, n_{k}$ are any integers satisfying $n_{i} \geq g(D)$, for $i=1,2, \ldots, k$ and $\sum_{i=1}^{k} n_{i}=n$ ?

By Theorem 13.4, $h(2)=2$. For all $k \geq 3$ the problem is open even for tournaments.

Conjecture 13.6 [42] $h(k)=f(k)$ for all $k$, where $f(k)$ is defined as in Problem 13.2.

\section{Miscellaneous results, problems and conjectures}

Jackson and Thomassen have the following conjecture [80].

Conjecture 14.1 [80] Every 2k-strongly connected digraph contains a spanning $k$-strongly connected oriented graph (i.e. a digraph without cycles of length two).

Even the weaker conjecture that there exists some function $f(k)$ such that every $f(k)$-strongly connected digraph contains a spanning $k$-strongly connected oriented graph is completely open for general digraphs. For locally semicomplete digraphs it is only known that $f(k) \leq 3 k-2[43](f(k) \leq 5 k$ was proved earlier in [6]). In [25] it is shown how this and Theorem 6.1 imply that the same $f(k)$ works for quasi-transitive digraphs as for semicomplete digraphs.

We believe that the following special cases of Conjecture 14.1 may be proved. It is however worth noting that even the very special case of semicomplete digraphs is open.

Conjecture 14.2 Every $2 k$-strongly connected semicomplete multipartite digraph contains a spanning $k$-strongly connected multipartite tournament.

Conjecture 14.3 Every $2 k$-strongly connected locally in-semicomplete digraph contains a spanning $k$-strongly connected locally in-tournament digraph.

Thomassen also posed the following conjecture for tournaments.

Conjecture 14.4 For all natural numbers $r, s$ there exists a natural number $f(r, s)$ such that the vertex set of every $f(r, s)$-strongly connected tournament $T$ can be partitioned into two sets $V_{1}, V_{2}$ such that the tournament induced by $V_{1}$ is r-strongly connected and the tournament induced by $V_{2}$ is s-strongly connected.

We believe that a similar property holds for quasi-transitive digraphs and locally semicomplete digraphs.

A kernel in a digraph $D=(V, A)$ is an independent set $X \subset V$ such that every vertex $y \in V-X$ is dominated by some vertex from $X$. Kernel is the problem of deciding whether a given digraph has a kernel.

Conjecture 14.5 Kernel is polynomially solvable for locally in-semicomplete digraphs .

Kernel is polynomially solvable for locally semicomplete digraphs [11]. It is easy to see that Kernel is polynomially solvable for semicomplete multipartite digraphs, since any kernel must be a subset of a colour class of the semicomplete multipartite digraphs. 
Problem 14.6 Determine the complexity of Kernel for path-mergeable digraphs.

We finish this section by discussion of some results on radius and diameter of some generalizations of tournaments. The diameter of a strongly connected digraph $D$ is the least integer $d$ such that, for every ordered pair $x, y$ of distinct vertices of $D, D$ has an $(x, y)$-path of length at most $d$.

Let $f\left(m_{1}, \ldots, m_{k}\right)$ be the minimum possible diameter of a $k$-partite tournament with partite sets of sizes $m_{1}, \ldots, m_{k}$. For $k=2$ the value of this function was determined by Soltes (for details see [55]). For $k \geq 3$ the function was investigated independently in [53, 65, 73]. It is easy to show that $2 \leq f\left(m_{1}, \ldots, m_{k}\right) \leq 3$ for every $k \geq 3$ and all positive integers $m_{1}, \ldots, m_{k}$ (see $[53,65,73]$ ). The following natural question was posed in [73] and [53]: Determine all $k$ tuples of integers $m_{1}, \ldots, m_{k}$ such that $f\left(m_{1}, \ldots, m_{k}\right)=2$. In $[53,65,73]$, it was shown that $f\left(m_{1}, \ldots, m_{k}\right)=2$ for all $m_{1}=m_{2}=\ldots=m_{k}$ except $m_{1}=m_{2}=m_{3}=m_{4}=1$. This result was extended in [66].

A pair $p, q$ of integers is called a co-pair if $1 \leq p \leq q \leq\left(\begin{array}{c}p \\ \lfloor p / 2\rfloor\end{array}\right)$. A triple $p, q, r$ of positive integers is called a co-triple if $p, q$ and $p, r$ are co-pairs. The following theorem is due to Koh and Tan [66]

Theorem 14.7 If $m_{1}, \ldots, m_{k}$ can be partitioned into co-pairs when $k$ is even and into co-pairs and a co-triple when $k$ is odd, then $f\left(m_{1}, \ldots, m_{k}\right)=2$.

The following notion is closely related to the notion of radius. A vertex $x$ of a digraph $D$ is a $r$-king if for every vertex $y \neq x$ there exists an $(x, y)$-path of length at most $r$. It is well known that every tournament has a 2-king. The first result on kings in multipartite tournaments was obtained independently in [50] and [72]: every multipartite tournament $T$ with at most one vertex of indegree zero has a 4-king (i.e. the radius of $T$ is at most four). This result was strengthened by Koh and Tan $($ see $[67,75])$ :

Theorem 14.8 Every $k$-partite tournaments without vertices of indegree zero contains at least four 4-kings when $k=2$ and at least three 4 -kings when $k \geq 3$.

The case of $k=2$ in Theorem 14.8 was independently proved in [48] and [71]. Moreover, this case is a simple consequence of the following result.

Theorem 14.9 [52] Let $T$ be a multipartite tournament. If $T$ has no vertex $x$ dominating all vertices of a partite set of $T$, then every partite set of $T$ has at least two 4-kings.

Note that there exist infinitely many $k$-partite tournaments without 3-kings for every $k \geq 2$ [50]. Some further results on $r$-kings in multipartite tournaments are well described in [75].

Bang-Jensen and Huang [26] considered kings in quasi-transitive digraphs. The main result of [26] is the following:

Theorem 14.10 A quasi-transitive digraph $D$ has a 3-king if and only if it has a finite radius. Furthermore, if $D$ has a 3-king, then every vertex in $D$ of maximum out-degree is a 3-king.

Note that there exist infinitely many quasi-transitive digraphs with 3-kings but without 2-kings.

\section{References}

[1] B. Alspach and C. Tabib, A note on the number of 4-circuits in a tournament, Annals of Disc. Math. 12 (1982) 13-19. 
[2] H. Alt, N. Blum, K. Melhorn and M. Paul, Computing of maximum cardinality matching in a bipartite graph in time $O\left(n^{1.5} \sqrt{m / \log n}\right)$. Inf. Proc. Letters 37 (1991) 237-240.

[3] J. Bang-Jensen, Arc-local tournament digraphs: a generalization of tournaments and bipartite tournaments. Preprint Department of Math. and CS Odense Univ., no. 2 (1993)

[4] J. Bang-Jensen, A note on special case of the 2-path problem for semicomplete digraphs, in "Conference Proc. of the 6th Intern. Conference on Graph Theory and Applications", Kalamazoo, 1988, 77-86.

[5] J. Bang - Jensen, Edge-disjoint in- and out-branchings in tournaments and related path problems, J. Combinatorial Theory, B 51 (1991) 1 - 23.

[6] J. Bang-Jensen, Locally semicomplete digraphs: a generalization of tournaments, J. Graph Theory 14 (1990) 371-390.

[7] J. Bang-Jensen, On the structure of locally semicomplete digraphs, Discrete Math. 100 (1992) 1-23.

[8] J. Bang-Jensen, On the 2-linkage problem for semicomplete digraphs. Annals of Disc. Math. 41 (1989) 23-38.

[9] J. Bang-Jensen, Digraphs with the path-merging property, J. Graph Theory 20 (1995) 255-265.

[10] J. Bang-Jensen, Disjoint paths with prescribed ends and cycles through given arcs in locally semicomplete digraphs and quasi-transitive digraphs, (1996) submitted.

[11] J. Bang-Jensen, Y. Guo, G. Gutin and L. Volkmann, A classification of locally semicomplete digraphs. 15th British Combinatorial Conference (Stirling, 1995). Discrete Math. 167/168 (1997) 101-114..

[12] J. Bang-Jensen, Y. Guo and L. Volkmann, Weakly Hamiltonian-connected locally semicomplete digraphs, J. Graph Theory 21 (1996) 163-172.

[13] J. Bang-Jensen and G. Gutin, Finding maximum weight paths and cycles in $\Phi$-decomposable digraphs, using flows in networks. Preprint Department of Math. and CS Odense Univ., no. 51 (1993)

[14] J. Bang-Jensen and G. Gutin, Paths and cycles in extended and decomposable digraphs. The Second Krakow Conference on Graph Theory (Zgorzelisko, 1994). Discrete Math. 164 (1997) 5-19.

[15] J. Bang-Jensen and G. Gutin, Longest paths and cycles in extended locally semicomplete digraphs, Preprint Department of Math. and CS Odense Univ., no. 53 (1993).

[16] J. Bang-Jensen and G. Gutin, Paths, trees and cycles in tournaments, Congressus Nummerantium 115 (1996) 131170 .

[17] J. Bang-Jensen and G. Gutin, Vertex heaviest paths and cycles in quasi-transitive digraphs, Discrete Math. 163 (1997) 217-223.

[18] J. Bang-Jensen, G. Gutin, and J. Huang, Weekly hamiltonian-connected ordinary multipartite tournaments. Discrete Math. 138 (1995) 63-74.

[19] J. Bang-Jensen, G. Gutin, and J. Huang, A sufficient condition for a complete multipartite digraph to be Hamiltonian, Discrete Math. 161 (1996) 1-12.

[20] J. Bang-Jensen, G. Gutin and A. Yeo, Hamiltonian cycles avoiding prescribed arcs in tournaments, Combinatorics, Probability and Computing 6 (1997) 255-261.

[21] J. Bang-Jensen, G. Gutin and A. Yeo, On k-strong and k-cyclic digraphs, Discrete Math. 162 (1996) 1-11.

[22] J. Bang-Jensen, G. Gutin and A. Yeo, A polynomial algorithm for the Hamiltonian cycle problem in semicomplete multipartite digraphs, submitted.

[23] J. Bang-Jensen and P. Hell, Fast algorithms for finding hamiltonian paths and cycles in in-tournament digraphs. Discrete Appl. Math. 41 (1991) 75-79.

[24] J. Bang-Jensen, P. Hell and J. Huang, Optimal recognition of local tournaments, Congressus Nummerantium 100 (1994) 141-146.

[25] J. Bang -Jensen and J. Huang, Quasi-transitive digraphs, J. Graph Theory 20 (1995) 141-161.

[26] J. Bang -Jensen and J. Huang, Kings in quasi-transitive digraphs, Discrete Math., to appear.

[27] J. Bang-Jensen, J. Huang and E. Prisner, In-tournaments. J. Combin. Theory B 59 (1993) 267-287.

[28] J. Bang-Jensen and Y. Manoussakis, Weakly hamiltonian-connected vertices in bipartite tournaments, J. Combin. Theory B 63 (1995) 261-280.

[29] J. Bang-Jensen, Y. Manoussakis and C. Thomassen, A polynomial algorithm for hamiltonian-connectedness in semicomplete digraphs. J. Algorithms 13 (1992) 114-127.

[30] J. Bang-Jensen and C. Thomassen, A polynomial algorithm for the 2-path problem for semicomplete digraphs. SIAM J. Discrete Math. 5 (1992) 366-376.

[31] Y. H. Bu and K. M. Zhang, On the arc-pancyclicity of local tournaments, Ars Combin. 47 (1997) $242-254$.

[32] Y. H. Bu and K. M. Zhang, Completely strong path-connectivity of local tournaments, submitted.

[33] G. Chartrand and L. Lesniak, Graphs \& Digraphs, 3rd ed., Chapman and Hall, London, 1996. 
[34] X. Deng, P. Hell, and J. Huang, Recognition and representation of proper circular-arc graphs, Integer Programming and Combinatorial Optimization, Proceeding of the second IPCO conference, (Egon Balas, G. Cornuéjols, and R. Kannan, eds.) (1992) pp. 114 - 121.

[35] X. Deng, P. Hell, and J. Huang, Linear time representation algorithms for proper circular-arc graphs and proper interval graphs, Siam J. Comput. 25 (1996) 390-403.

[36] S. Fortune, J. Hopcroft and J. Wyllie, The directed subgraph homomorphism problem, Theoret. Comput. Sci. 10 (1980), 111-121.

[37] T. Gallai, Transitiv orientierbarer graphen, Acta Mathematica Acad. Sci. Hungaricae 18 (1967) 25-66.

[38] A. Ghouilà-Houri, Caractérisation des graphes non orientès dont on peut orienter les arrêtes de maniere à obtenir le graphe d'un relation d'ordre. C. R. Acad. Sci. Paris 254 (1962) 1370 - 1371.

[39] M.C.Golumbic, Algorithmic Graph Theory and perfect graphs, Academic Press, New York (1980).

[40] Y. Guo, Bypaths in Local tournaments. 4th Twente Workshop on Graphs and Combinatorial Optimization (Enschede, 1995). Discrete Appl. Math. 79 (1997) 127-135.

[41] Y. Guo, Path-connectivity in local tournaments. 5th British Combinatorial Conference (Stirling, 1995). Discrete Math. 167/168 (1997) 353-372.

[42] Y. Guo, Locally semicomplete digraphs, Ph.D. thesis, RTWH Aachen, Germany (1995).

[43] Y. Guo, Spanning locally tournament digraphs in locally semicomplete digraphs. 4th Twente Workshop on Graphs and Combinatorial Optimization (Enschede, 1995). Discrete Appl. Math. 79 (1997) 119-125.

[44] Y. Guo, Strongly Hamiltonian-connected locally semicomplete digraphs, J. Graph Theory, 22 (1996) 65-73.

[45] Y. Guo and L. Volkmann, Connectivity properties of locally semicomplete digraphs, J. Graph Theory 18 (1994) 269-280.

[46] Y. Guo and L. Volkmann, On complementary cycles in locally semicomplete digraphs, Dicrete Math. 135 (1994) $121-127$.

[47] Y. Guo and L. Volkmann, Locally semicomplete digraphs that are complementary m-pancyclic, J. Graph Theory 21 (1996) 121-136.

[48] G. Gutin, On bipartite tournaments. Dep. in VINITI, no. 5275 (1982) 9 p.

[49] G. Gutin, A criterion for complete bipartite digraphs to be Hamiltonian. Vestsi Acad. Navuk BSSR Ser. Fiz.-Mat. Navuk No. 1 (1984) 99-100 (In Russian).

[50] G. Gutin, The radii of $n$-partite tournaments. Math. Notes 40 (1986) 743-744.

[51] G. Gutin, A characterization of complete n-partite digraphs that have a Hamiltonian path. Kibernetica No. 1 (1988) 107-108 (In Russian).

[52] G. Gutin, m-sources in complete multipartite digraphs, Vestsi Acad. Navuk BSSR Ser. Fiz.-Mat. no. 5 (1989) 101-106 (in Russian).

[53] G. Gutin, Minimizing and maximizing the diameter in orientations of graphs, Graphs Comb. 10 (1994) 225-230.

[54] G. Gutin, Finding a longest path in a complete multipartite digraph, SIAM J. Disc. Math. 6 (1993) 270-273.

[55] G. Gutin, Cycles and paths in semicomplete multipartite digraphs, Theorems and algorithms: A survey, J. Graph Theory 19 (1995) 481-505.

[56] G. Gutin, Paths and Cycles in Directed graphs, Ph.D. Thesis, Tel-Aviv University (1993).

[57] G. Gutin, Characterizations of vertex pancyclic and pancyclic ordinary complete multipartite digraphs, Discrete Mathematics 141 (1995) 153-162.

[58] G. Gutin, Polynomial algorithms for finding hamiltonian paths and cycles in quasi-transitive digraphs, Australasian J. Combin. 10 (1994) 231-236.

[59] R. Häggkvist, Hamiltonian cycles in oriented graphs, Combinatorics, Probability and Computing 2 (1993) 25-32.

[60] R. Häggkvist and Y. Manoussakis, Cycles and paths in bipartite tournaments with spanning configurations. Combinatorica 9 (1989) 33-38.

[61] P. Hell, J. Bang-Jensen and J. Huang, Local tournaments and proper circular-arc graphs, Springer Lecture Notes in Computer Science 450 (1990) 101-108.

[62] P. Hell and J. Huang, Lexicographic orientation and representation algorithms for comparability graphs, proper circular-arc graphs, and proper interval graphs, J. Graph Theory 20 (1995) 361-374.

[63] J. Huang, On the structure of local tournaments, J. Combinatorial Theory B 63 (1995) 200-221.

[64] J. Huang, Tournament-like oriented graphs, Ph.D. thesis, Simon Fraser University, 1992.

[65] K.M. Koh and B.P. Tan, The diameter of an orientation of a complete multipartite graph, Discrete Math. 149 (1996) 131-139. 
[66] K.M. Koh and B.P. Tan, The minimum diameter of orientations of complete multipartite graphs, Graphs and Combinatorics 12 (1996) 333-339.

[67] K.M. Koh and B.P. Tan, Kings in multipartite tournaments. Discrete Math. 147 (1995) 171-183.

[68] Manoussakis Y., A linear time algorithm for finding Hamiltonian cycle in tournaments. Discrete Appl. Math. 36 (1992) 199-202.

[69] Y. Manoussakis and Z. Tuza, Polynomial algorithms for finding cycles and paths in bipartite tournaments. SIAM J. Disc. Math. 3 (1990) 537-543.

[70] H. Papadimitriou and K. Steiglitz, Combinatorial Optimization: Algorithms and Complexity. Prentice-Hall Inc., New Jersey, 1982.

[71] V. Petrovic, Kings in bipartite tournaments, Discrete Math. 173 (1997) 187-196.

[72] V. Petrovic and C. Thomassen, Kings in k-partite tournaments. Discrete Math. 98 (1991) 237-238.

[73] J. Plesnik, Remarks on diameters of orientations of graphs, Acta Math. Univ. Comenianae 46/47 (1985) 225-236.

[74] K.B. Reid, Two complementary circuits in two-connected tournaments. Ann. Discrete Math. 27 (1985) 321-334.

[75] K.B. Reid, Tournaments: scores, kings, generalizations and special topics. Congressus Nummerantium 115 (1996) 171-211.

[76] D.J. Skrien, A relationship between triangulated graphs, comparability graphs, proper interval graphs, proper circulararc graphs and nested interval graphs, J. Graph Theory, 6 (1980) 309 - 316

[77] Z.M. Song, Complementary cycles of all lengths in tournaments. J. Combin. Theory Ser. B 57 (1993) 18-25.

[78] C. Thomassen, Hamiltonian-connected tournaments, J. Combinatorial Theory B 28 (1980) 142 - 163.

[79] C. Thomassen, Connectivity in tournaments. In Graph Theory and Combinatorics, B. Bollobás, ed., Acad. Press (1984) 305-313.

[80] C. Thomassen, Configurations in graphs of large minimum degree, connectivity, or chromatic number, Annals of the New York Academy of Sciences 555 (1989) 402-412.

[81] J. Urrutia and F. Gavril, An algorithm for fraternal orientation of graphs, Inform. Process. Lett. 41 (1992) $271-274$.

[82] L. Volkman, a talk at The Second Krakow Conference of Graph Theory, 1994.

[83] L. Volkmann, Fundamente der Graphentheorie, Springler-Verlag, Wien New Yourk, 1996.

[84] L. Volkmann, Cycles in multipartite tournaments: results and problems, submitted.

[85] A. Yeo, One-diregular subgraphs in semicomplete multipartite digraphs, Journal of Graph Theory 24 (1997) 1-11.

[86] C. Q. Zhang, Hamilton paths in multipartite oriented graphs, Ann. Discrete Math. 41 (1989) 499-514.

[87] C. Q. Zhang and C. Zhao, On locally semicomplete digraphs, Journal of Combinatorial Math. and Combinatorial Computing 20 (1996) 186-192.

[88] H. Zhou and X. Zhu, Multiplicativity of acyclic local tournaments, Combinatorica 17 (1997) 135-145. 